\title{
Etude de l'écoulement et de la répartition des pressions au voisinage du piston d'un amortisseur hydraulique
}

\author{
$\mathrm{Ph}$. Le et $\mathrm{R}$. Comolet \\ Laboratoire de Mécanique des Fluides, U.A. 871, Bâtiment 502, Campus Universitaire, 91405 Orsay, France
}

(Reçu le 18 juin 1987, révisé le 16 octobre 1987, accepté le 20 mars 1989)

\begin{abstract}
Résumé. - Nous établissons un critère permettant de caractériser l'écoulement dans un amortisseur hydraulique. Pour cela, nous appliquons l'analyse phénoménologique aux équations de Navier-Stokes et parvenons à montrer que, selon la valeur du gradient de pression, il existe deux régimes d'écoulement, malgré les faibles nombres de Reynolds. On en déduit que les pertes de charge se produisent tantôt sous forme visqueuse, tantôt sous forme inertielle. Un modèle basé sur l'analogie entre un écoulement radial divergent et l'écoulement se produisant sous le piston, nous permet d'évaluer la répartition des pressions puis l'expression de la force résultante qui s'exerce à ce niveau. Les prévisions de l'analyse phénoménologique concordent avec les résultats établis par les essais expérimentaux simulant l'écoulement.
\end{abstract}

\begin{abstract}
A criterion characterizing the flow in an oil damper is established. A phenomenological analysis is applied, based on the Navier-Stokes equations and show that, there are two regimes of flow in fonction of the pressure gradient values, in spite of the low Reynolds numbers involve. The losses of pressure occur either under viscous form, or under inertial form. A model based on the analogy between a radial divergent flow and a flow taking place under the piston, allows to evaluate quantitatively the pressure distribution and the expression of the resulting force at the piston level. The predictions of the phenomenological analysis are confirmed by experiments simulating the flow in the vicinity of the piston.
\end{abstract}

\section{Introduction.}

Amortir les vibrations subies par les véhicules est un problème qui se pose à partir d'une certaine vitesse et pour certains profils routiers.

Ces vibrations produisent des effets néfastes tant sur le plan humain que sur le plan matériel. Ces effets indésirables se traduisent par une diminution du confort, une dégradation de la tenue de route et une usure prématurée du véhicule $[1,2]$.

Afin de remédier à cette situation, des éléments déformables constituant la suspension et dont font partie les amortisseurs ont été introduits [3].

\section{Description.}

Comme le montre la figure 1, un amortisseur hydraulique de type monotube se compose de deux chambres de travail cylindrique $\mathrm{A}$ et $\mathrm{B}$, remplies d'huile dans laquelle peut coulisser un système tigepiston-clapet. L'huile peut passer d'une chambre à l'autre par l'intermédiaire d'orifices calibrés pratiqués dans le piston en soulevant l'un des deux clapets situés de part et d'autre de ce dernier. Les pertes de charge provoquées par le mouvement de l'huile sont localisées principalement dans l'entrefer piston-clapet. L'étude que nous entreprenons concerne donc l'écoulement à ce niveau [4-6]

\section{Modèle phénoménologique de l'écoulement.}

Nous nous proposons de simplifier les équations de Navier-Stokes en déterminant l'ordre de grandeur des différents termes de ces équations et de n'y retenir que ceux d'ordre supérieur.

\subsection{HyPOTHÈSES. - Nous supposerons que :}

- l'huile est incompressible, isotrope et son comportement est newtonien. Sa viscosité ne dépend pas de la pression;

- les effets dus à la gravité et à la tension superficielle sont négligeables ; 


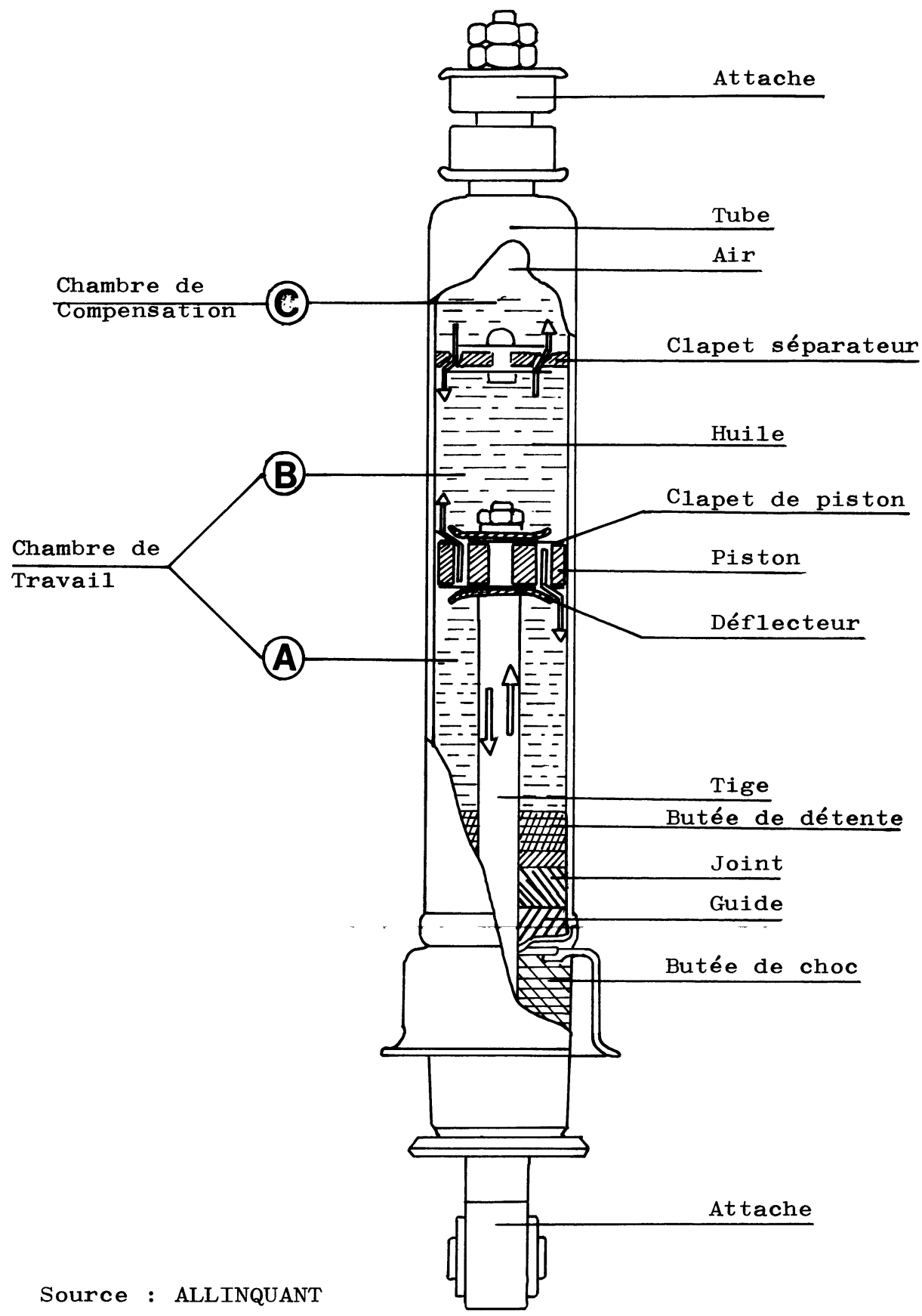

Fig. 1. - Principe de fonctionnement de l'amortisseur monotube.

[Monotube shock absorber. Operating principle.]

- le processus est isotherme ;

- le régime d'écoulement peut toujours être considéré comme laminaire car les nombres de Reynolds sont faibles pour les pressions utilisées;

- nous négligerons la déformation du cylindre en fonction de la pression, étant donné son épaisseur devant les dimensions de la chambre intérieure et la nature du matériau dont il est constitué ;

- nous supposerons que les conditions d'entrée sont telles que l'écoulement est à pression constante avant l'entrefer (les pertes de charge régulières et singulières sont négligeables avant et dans le piston), et qu'en raison des faibles nombres de Reynolds (calculés ultérieurement), l'écoulement est établi dans l'entrefer piston-clapet.

3.2 EQUATIONS. - Choisissons un repère cylindrique centré sur un orifice de passage d'huile (Fig. 2) et posons comme grandeurs caractéristiques :

$n$ : nombre d'orifices du clapet amont, $w_{0}$ : vitesse moyenne dans un orifice, $r_{0}$ : rayon d'un orifice, 


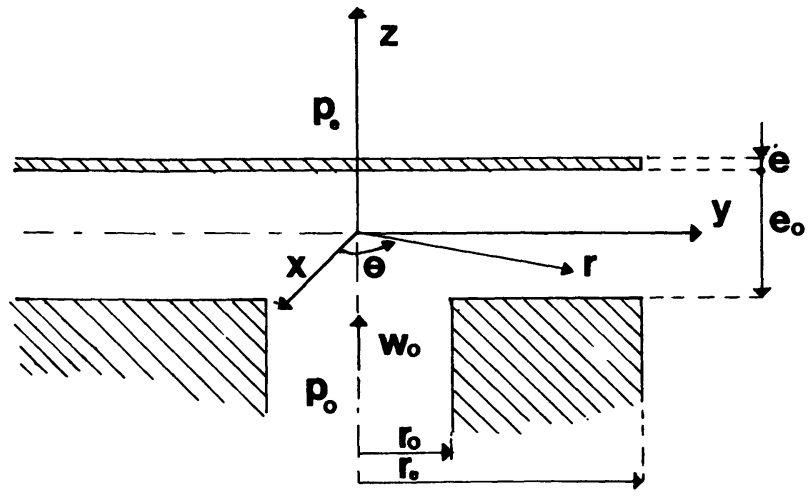

Fig. 2. - Entrefer clapet-piston.

[Piston valve clearance.]

$r_{\mathrm{cl}}:$ rayon du clapet,

$e_{0}$ : hauteur de l'entrefer piston-clapet,

$e$ : épaisseur du clapet,

$P_{\mathrm{e}}$ : pression extérieure,

$\Delta P$ : gradient de pression entre l'entrée et la sortie de l'entrefer,

$Q_{\mathrm{v}}$ : débit volumique d'huile.

Nous supposerons que la vitesse tangentielle $v$ est peu différente de la vitesse radiale $u$.
Comme le débit qui rentre par les orifices est égal à celui qui sort par l'entrefer, nous avons la relation :

$$
Q_{\mathrm{v}}=n \pi r_{0}^{2} w_{0}=2 \pi r_{\mathrm{cl}} e_{0} u\left(r_{\mathrm{cl}}\right) \text {. }
$$

Posons $\varepsilon=e_{0} / r_{0}, K=n r_{0}^{2} / 2 \cdot r_{\mathrm{cl}} e_{0}$ et définissons les variables réduites :

$$
\begin{aligned}
u^{+} & =u / K w_{0}, \\
v^{+} & =v / K w_{0}, \\
P^{+} & =\left(P-P_{\mathrm{e}}\right) / \Delta P \\
r^{+} & =r / r_{0} \\
z^{+} & =z / e_{0} .
\end{aligned}
$$

Nous définirons $w^{+}$à partir de l'équation de continuité :

$$
\frac{K w_{0}}{r_{0}} \frac{\partial r^{+} u^{+}}{r^{+} \partial r^{+}}+\frac{K w_{0}}{r_{0}} \frac{\partial v^{+}}{\partial \theta}+\frac{\partial w}{e_{0} \partial z^{+}}=0 .
$$

Soit encore :

$$
\frac{\partial r^{+} u^{+}}{r^{+} \partial r^{+}}+\frac{\partial v^{+}}{\partial \theta}+\frac{r_{0}}{K w_{0} e_{0}} \frac{\partial w}{\partial z^{+}}=0
$$

Afin que tous les termes de cette expression soient d'ordre 1, choisissons $w^{+}=w r_{0} / K w_{0} e_{0}$.

Les équations de Navier-Stokes sous forme adimensionnelle, dans ce système d'axes, peuvent alors s'écrire :

$$
\begin{aligned}
& \frac{K^{2} w_{0}^{2}}{r_{0}} u^{+} \frac{\partial u^{+}}{\partial r^{+}}+\frac{K^{2} w_{0}^{2}}{r_{0}} \frac{v^{+}}{r^{+}} \frac{\partial u^{+}}{\partial \theta}-\frac{K^{2} w_{0}^{2}}{r_{0}} \frac{v^{+2}}{r^{+}}+\frac{K^{2} w_{0}^{2}}{r_{0}} w^{+} \frac{\partial u^{+}}{\partial z^{+}}=-\frac{\Delta P}{\rho r_{0}} \frac{\partial P^{+}}{\partial r^{+}}+ \\
& +\nu\left[\frac{K w_{0}}{r_{0}^{2}} \frac{\partial}{\partial r^{+}}\left[\frac{1}{r^{+}} \frac{\partial\left(r^{+} u^{+}\right)}{\partial r^{+}}\right]+\frac{K w_{0}}{e_{0}^{2}} \frac{\partial^{2} u^{+}}{\partial z^{+2}}+\frac{K w_{0}}{r_{0}^{2} r^{+}} \frac{\partial^{2} v^{+}}{\partial \theta^{2}}-\frac{2 K w_{0}}{r_{0}^{2}} \frac{1}{r^{+2}} \frac{\partial v^{+}}{\partial \theta}\right] \\
& \frac{K^{2} w_{0}^{2}}{r_{0}} u^{+} \frac{\partial v^{+}}{\partial r^{+}}+\frac{K^{2} w_{0}^{2}}{r_{0}} \frac{v^{+}}{r^{+}} \frac{\partial v^{+}}{\partial \theta}+\frac{K^{2} w_{0}^{2}}{r_{0}} w^{+} \frac{\partial v^{+}}{\partial z^{+}}+\frac{K^{2} w_{0}^{2}}{r_{0}} \frac{u^{+} v^{+}}{r^{+}}=-\frac{\Delta P}{\rho r_{0}} \frac{\partial P^{+}}{r^{+} y \theta}+ \\
& +\nu\left[\frac{K w_{0}}{r_{0}^{2}} \frac{\partial}{\partial r^{+}}\left[\frac{1}{r^{+}} \frac{\partial\left(r^{+} v^{+}\right)}{\partial r^{+}}\right]+\frac{K w_{0}}{r_{0}^{2}} \frac{\partial^{2} v^{+}}{r^{+2} \partial \theta^{2}}+\frac{K w_{0}}{e_{0}^{2}} \frac{\partial^{2} v^{+}}{\partial z^{+2}}+\frac{2 K w_{0}}{r_{0}^{2}} \frac{\partial u^{+}}{r^{+2} \partial \theta}\right] \\
& \frac{K^{2} w_{0}^{2} e_{0}}{r_{0}^{2}} u^{+} \frac{\partial w^{+}}{\partial r^{+}}+\frac{K^{2} w_{0}^{2} e_{0}}{r_{0}^{2}} \frac{v^{+}}{r^{+}} \frac{\partial w^{+}}{\partial \theta}+\frac{K^{2} w_{0}^{2} e_{0}}{r_{0}^{2}} w^{+} \frac{\partial w^{+}}{\partial z^{+}}=-\frac{\Delta P}{\rho e_{0}} \frac{\partial P^{+}}{\partial z^{+}}+ \\
& +\nu\left[\frac{K w_{0} e_{0}}{r_{0}^{3}} \frac{\partial^{2} w^{+}}{\partial r^{+2}}+\frac{K w_{0} e_{0}}{r_{0}^{3}} \frac{\partial w^{+}}{r^{+} \partial r^{+}}+\frac{K w_{0} e_{0}}{r_{0}^{3}} \frac{\partial^{2} w^{+}}{r^{+2} \partial \theta^{2}}+\frac{K w_{0}}{r_{0} e_{0}} \frac{\partial^{2} w^{+}}{\partial z^{+2}}\right] \text {. }
\end{aligned}
$$

Posons :

$$
\begin{array}{ll}
R_{\mathrm{p}}=\Delta P / \rho K^{2} w_{0}^{2}, & \text { terme caractérisant la pression }, \\
R_{\mathrm{v}}=\nu r_{0} / K e_{0}^{2} w_{0}, & \text { terme caractérisant la viscosité }, \\
\operatorname{Re}=Q_{\mathrm{v}} / 2 \pi \nu r_{\mathrm{cl}}, & \text { nombre de Reynolds local de l'écoulement }
\end{array}
$$


Nous obtenons alors, pour le système précédent les ordres de grandeur suivants :

$\begin{array}{ccc}\text { termes } & \text { termes } & \text { termes } \\ \text { d'inertie } & \text { de pression } & \text { de viscosité }\end{array}$

$\begin{array}{ccc}1 & R_{\mathrm{p}} & R_{\mathrm{v}}, \varepsilon^{2} R_{\mathrm{v}} \\ 1 & R_{\mathrm{p}} & R_{\mathrm{v}}, \varepsilon^{2} R_{\mathrm{v}} \\ \varepsilon & R_{\mathrm{p}} / \varepsilon & \varepsilon R_{\mathrm{v}}, \varepsilon^{3} R_{\mathrm{v}} .\end{array}$

3.3 DisCUSSION. - Si nous considérons l'équation (8), nous voyons que les termes de viscosité et d'inertie sont négligeables devant la pression. Celleci étant le moteur de l'écoulement, elle doit nécessairement rester dans l'équation. Les variations de $p$ selon $z$ sont donc d'ordre $\varepsilon^{2}$.

$\mathrm{Si}$ nous considérons maintenant l'ensemble du système, nous constatons que tous les termes de la $3^{\mathrm{e}}$ équation sont au plus d'ordre $\varepsilon$ par rapport aux deux premières équations. Nous pouvons donc négliger les phénomènes se produisant selon cet axe et traiter l'écoulement de façon bidimensionnelle.

Etudions l'importance de $R_{\mathrm{v}}$ et $R_{\mathrm{p}}$ par rapport à 1 :

a) $R_{\mathrm{v}} \gg 1$ et $R_{\mathrm{p}} \sim R_{\mathrm{v}}$ : les pertes de charges à ce niveau sont dues à la viscosité. L'huile est laminée dans l'entrefer ;

b) $R_{\mathrm{v}} \ll 1$ et $R_{\mathrm{p}} \sim 1$ : l'écoulement est dominé par l'inertie du fluide. La pression statique se transforme en pression dynamique. Elle-même sera perdue ultérieurement sous forme visqueuse à la sortie de l'entrefer ;

c) entre ces deux cas existe une zone où les trois termes adimensionnels sont de l'ordre de grandeur de l'unité : $R_{\mathrm{p}} \sim R_{\mathrm{v}} \sim 1$. C'est une zone de transition où l'influence de la viscosité est à peu près égale à celle de l'inertie ;

d) lorsque $e_{0} / r_{0} \sim 1$, l'écoulement est tridimensionnel. Cependant, ce cas est purement théorique dans notre problème puisque la flèche maximale des clapets ne dépasse pas $200 \mu\left(e_{0} / r_{0}=0,1\right)$.

\section{Calcul des pressions.}

Nous ferons l'analogie entre ce type d'écoulement et l'écoulement radial divergent qui se produit entre deux disques parallèles en calculant expérimentalement les coefficients de conversion [8-12].

Les premières tentatives pour tenir compte des termes d'accélération sont celles de Mac Ginn et de Comolet. Dans les deux cas, le profil des vitesses entre les deux disques est considéré comme étant parabolique :

$$
u / U=1-(2 z / h)^{2}
$$

$U$ étant la vitesse débitante de l'écoulement.

Mac Ginn prend une combinaison linéaire empirique de la répartition des pressions telle qu'elle est donnée par les équations des fluides parfaits et visqueux et propose l'expression suivante :

$$
\begin{aligned}
P(r)-P_{0}=-\frac{3 \mu Q_{\mathrm{v}}}{4 \pi h^{3}} & \ln \left(r / r_{0}\right)- \\
& -\frac{27 \rho Q_{\mathrm{v}}^{2}}{560 \pi^{2} h^{2}}\left[\frac{1}{r^{2}}-\frac{1}{r_{0}^{2}}\right] .
\end{aligned}
$$

La méthode proposée par Comolet est d'appliquer le théorème des quantités de mouvement sous forme intégrale. Il obtient :

$$
\begin{aligned}
P(r)-P_{0}=-\frac{3 \mu Q_{\mathrm{v}}}{4 \pi h^{3}} & \ln \left(r / r_{0}\right)- \\
& -\frac{3 \rho Q_{\mathrm{v}}^{2}}{80 \pi^{2} h^{2}}\left[\frac{1}{r^{2}}-\frac{1}{r_{0}^{2}}\right] .
\end{aligned}
$$

Moller fait intervenir une légère déformation du profil de vitesse en fonction de $r$. Il utilise la même méthode que Comolet mais en prenant une répartition de vitesse voisine d'une répartition parabolique contenant un terme dépendant de $r$ qu'il détermine en utilisant l'équation de continuité, soit :

$$
u / U=\frac{4 z}{h}\left(1-\frac{z}{h}\right)+\xi \frac{z}{h}\left(1-\frac{2 z}{h}\right)^{3} .
$$

Il arrive alors à la formule :

$$
\begin{aligned}
P(r)-P_{0}=-\frac{3 \mu Q_{\mathrm{v}}}{4 \pi h^{3}} & \ln \left(r / r_{0}\right)- \\
& -\frac{9 \rho Q_{\mathrm{v}}^{2}}{200 \pi^{2} h^{2}}\left(\frac{1}{r^{2}}-\frac{1}{r_{0}^{2}}\right) .
\end{aligned}
$$

Toutes ces études font ressortir une répartition de pression de la forme :

$$
P(r)-P_{0}=\frac{\alpha \mu Q_{\mathrm{v}}}{n h^{3}} \ln \frac{r}{r_{0}}+\frac{\beta \rho Q_{\mathrm{v}}^{2}}{n^{2} h^{2}}\left(\frac{1}{r^{2}}-\frac{1}{r_{0}^{2}}\right)
$$

où $\alpha$ et $\beta$ caractérisent respectivement le terme de viscosité et le terme d'inertie. Ce sont des constantes qui dépendent du profil de vitesse choisi par les auteurs précités.

Tableau I. - Coefficients théoriques $\alpha$ et $\beta$ pour différents profils de vitesse.

[Theoretical coefficients $\alpha$ and $\beta$ for various velocity profiles.]

\begin{tabular}{|l|c|c|}
\hline & $\begin{array}{c}\text { Terme } \\
\text { visqueux }\end{array}$ & $\begin{array}{c}\text { Terme } \\
\text { inertiel }\end{array}$ \\
\hline Mac Ginn & 0,24 & 0,0049 \\
Comolet & 0,24 & 0,0038 \\
Moller & 0,24 & 0,0046 \\
\hline
\end{tabular}


Sous forme adimensionnelle, l'équation (14) peut encore s'écrire :

$$
\frac{\Delta P h^{4} n^{2}}{1 / 2 \rho Q_{\mathrm{v}}^{2}}=\frac{2 \alpha \nu h n}{Q_{\mathrm{v}}} \ln \frac{r}{r_{0}}+2 \beta h^{2}\left(\frac{1}{r^{2}}-\frac{1}{r_{0}^{2}}\right) .
$$

Multiplions l'expression par $e^{2} / h^{2}$ et faisons intervenir le nombre de Reynolds local de l'écoulement, soit :

$$
\frac{\Delta P h^{2} e^{2} n^{2}}{1 / 2 \rho Q_{\mathrm{v}}^{2}}=\frac{\alpha n e^{2}}{\pi r_{\mathrm{cl}} h \cdot \operatorname{Re}} \ln \frac{r}{r_{0}}+2 \beta e^{2}\left(\frac{1}{r^{2}}-\frac{1}{r_{0}^{2}}\right) .
$$

En posant

$$
\begin{aligned}
X & =\frac{n e^{2}}{\pi h \operatorname{Re} R_{\mathrm{cl}},} \\
\text { et } \quad Y & =\frac{2 \Delta P h^{2} e^{2} n^{2}}{\rho Q_{\mathrm{v}}^{2}},
\end{aligned}
$$

nous obtenons alors une équation linéaire dont les caractéristiques $\alpha$ et $\beta$ peuvent être déterminées par régression. $h$ est calculé en choisissant la déformation maximale du clapet. Les coefficients $\alpha, \beta$ sont expérimentaux et seront calculés ultérieurement.

\section{Calcul des forces de pressions.}

La force qui s'exerce sur le clapet récepteur est calculée en intégrant l'expression (10) sur une surface que nous choisirons arbitrairement en forme de demi-couronne entourant les sources, de diamètre intérieur $r_{0}$ (rayon de la source) et du diamètre extérieur $r_{1}=3 \mathrm{~mm}$ (distance minimale à la périphérie du clapet) (Fig. 3). Cette surface a été définie en supposant que l'écoulement se fait préférentiellement vers l'extérieur de l'entrefer et non pas vers l'encastrement. Ce choix a été guidé par l'empreinte

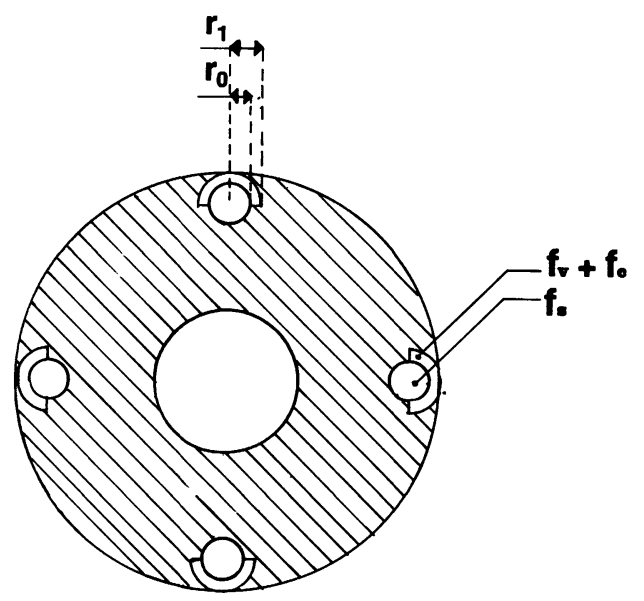

Fig. 3. - Aire de répartition des forces sur le clapet.

[Forces distribution areas on the valve.] des lignes de courant laissées par l'huile sous les clapets. Nous obtenons alors une expression de la forme :

$$
\begin{aligned}
F=\pi \int_{r_{0}}^{r_{1}}\left[\frac{\alpha \mu Q_{\mathrm{v}}}{n h^{3}}\right. & \cdot \ln \left(r_{0} / r\right)+ \\
& \left.+\frac{\rho \beta}{n^{2}}\left(\frac{1}{r_{0}^{2}}-\frac{1}{r^{2}}\right) \frac{Q_{\mathrm{v}}^{2}}{h^{2}}\right] r \mathrm{~d} r
\end{aligned}
$$

dont l'intégration conduit à une force d'origine visqueuse :

$$
F_{\mathrm{v}}=\pi \frac{\alpha \mu Q_{\mathrm{v}}}{n h^{3}}\left[\frac{r_{1}^{2}}{2}\left(\ln \left(r_{0} / r_{1}\right)+\frac{1}{2}\right)-\frac{r_{0}^{2}}{4}\right]
$$

et une force d'origine inertielle :

$$
F_{\mathrm{c}}=\pi \frac{\rho \beta Q_{\mathrm{v}}^{2}}{n^{2} h^{2}}\left(\frac{r_{1}^{2}}{2 r_{0}^{2}}-\frac{1}{2}-\ln \left(r_{1} / r_{0}\right)\right) .
$$

La force totale s'exerçant sur le clapet sera obtenue en ajoutant à ces deux forces, la force de pression statique issue de chaque source, multipliée par leur nombre.

$$
F_{\mathrm{t}}=n\left(\pi P_{0} r_{0}^{2}+F_{\mathrm{v}}+F_{\mathrm{c}}\right)
$$

\section{Essais expérimentaux.}

La figure 4 illustre l'ensemble du dispositif en régime statique. 7501 d'huile (MOBIL Infiltrex 650) sont comprimés dans un réservoir. Pour des raisons de commodités, cylindre et piston seront maintenus fixes l'un par rapport à l'autre. L'huile sera injectée sous la pression désirée. Nous admettons alors que les mouvements relatifs entre l'huile et l'amortisseur sont identiques, la pression étant ajustable. On en déduit que les écoulements sont analogues. Le dispositif se compose d'un cylindre dans lequel est placé un piston et deux clapets de part et d'autre.

Deux capteurs de pressions Sensotec à jauge de contrainte, $\mathrm{T} 1$ et $\mathrm{T} 2$ disposés en amont et en aval du piston permettent de connaître le gradient de pression à ce niveau. Un capteur de déplacement T3 Schlumberger à transformateur différentiel s'appuie sur la périphérie du clapet et permet de mesurer sa déformation sous l'effet de la pression [7].

Nous avons réalisé la partie expérimentale en interchangeant plusieurs types de clapet. Ceux-ci diffèrent par leur épaisseur et le nombre d'orifices qu'ils peuvent comporter (Fig. 5). Nous avons ainsi fait varier l'épaisseur du clapet aval et modifié le nombre d'orifices du clapet amont. Le premier paramètre a une influence sur les pertes de charge lorsqu'il affecte le clapet aval, tandis que le second agit sur le nombre de points de poussées de l'huile sous le clapet. 


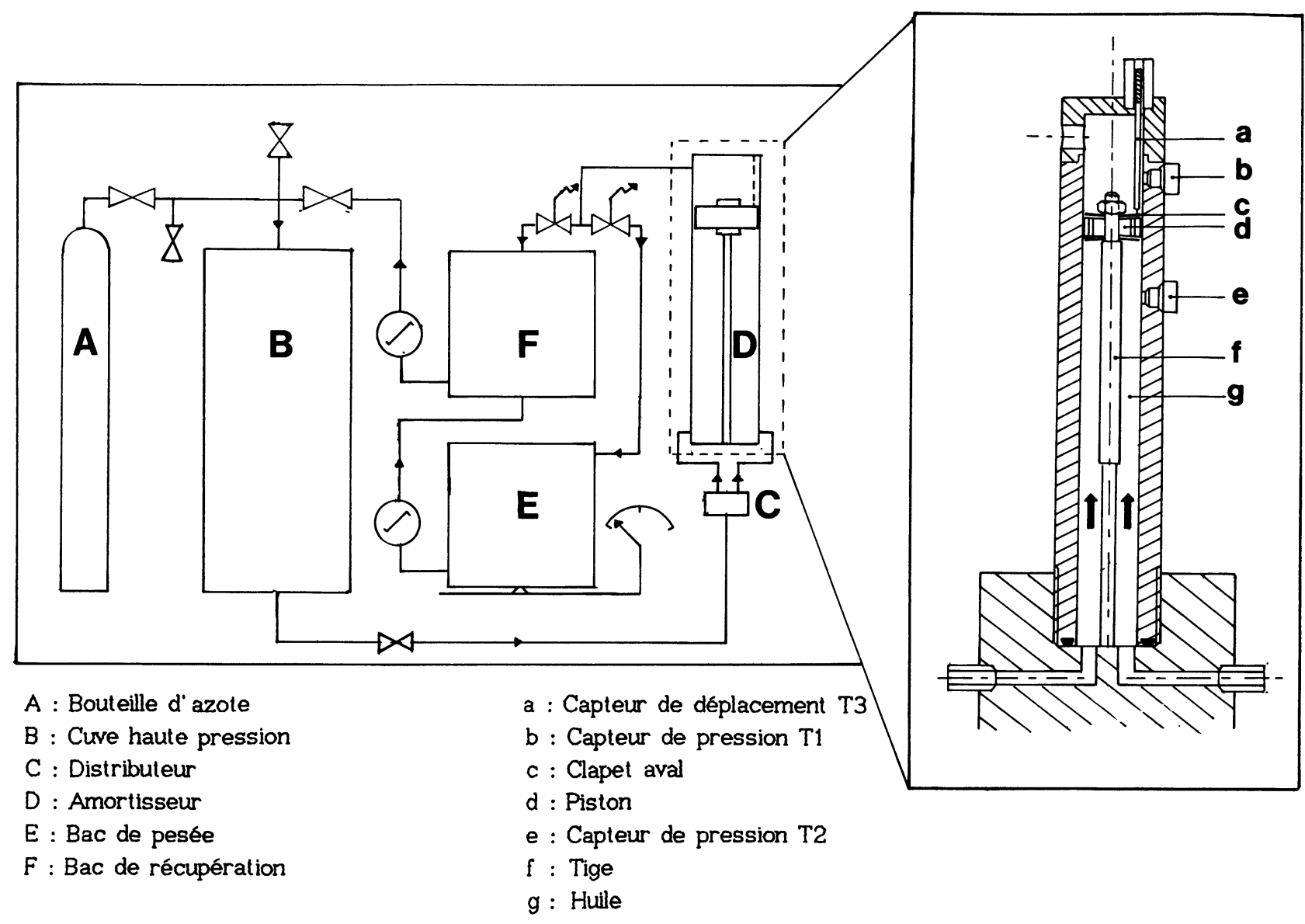

Fig. 4. - Plan synoptique de l'installation.

[Synoptic table of the test bench.]

Nous pouvons voir sur la figure 6, l'évolution du débit d'huile en fonction du gradient de pression. $Q_{\mathrm{v}}$ est mesuré par pesée. Cette courbe a été établie pour un clapet de $0,2 \mathrm{~mm}$ d'épaisseur, soulevé en quatre points répartis de façon symétrique et concentrique. D'une manière générale, lorsque l'épaisseur augmente, les courbes de débit/pression tendent à s'aplatir, les pertes de charge étant plus importantes.

La figure 7 montre l'évolution de la flèche du clapet précédent en fonction du même gradient de pression. Cette déformation est relevée en sept points d'azimuts bien précis, prédéfinis par un déflecteur surmontant le clapet.

Nous déduisons de cette série de courbes, des diagrammes périphériques (Fig. 8), d'allure sinusoïdale, dont la période correspond au nombre d'orifices du clapet amont et dont l'amplitude dépend à la fois de la pression et de l'épaisseur du clapet considéré.

Nous avons testé les épaisseurs de : $0,20,0,25$, $0,30,0,35,0,40,0,60 \mathrm{~mm}$ et dans le cas que nous traitons, le clapet aval est soulevé en 4 points, équivalent au nombre d'orifices du clapet amont.

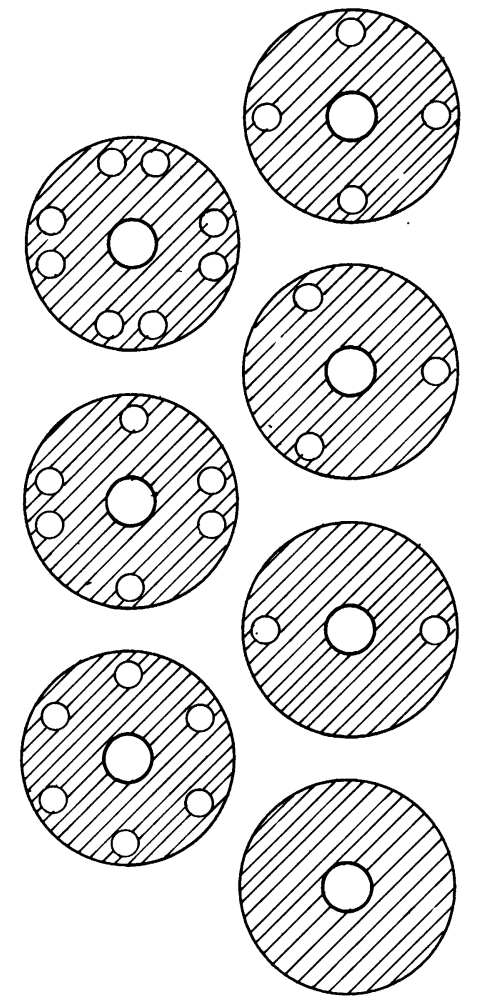

Fig. 5. - Différents types de clapets.

[Various kinds of valves.] 


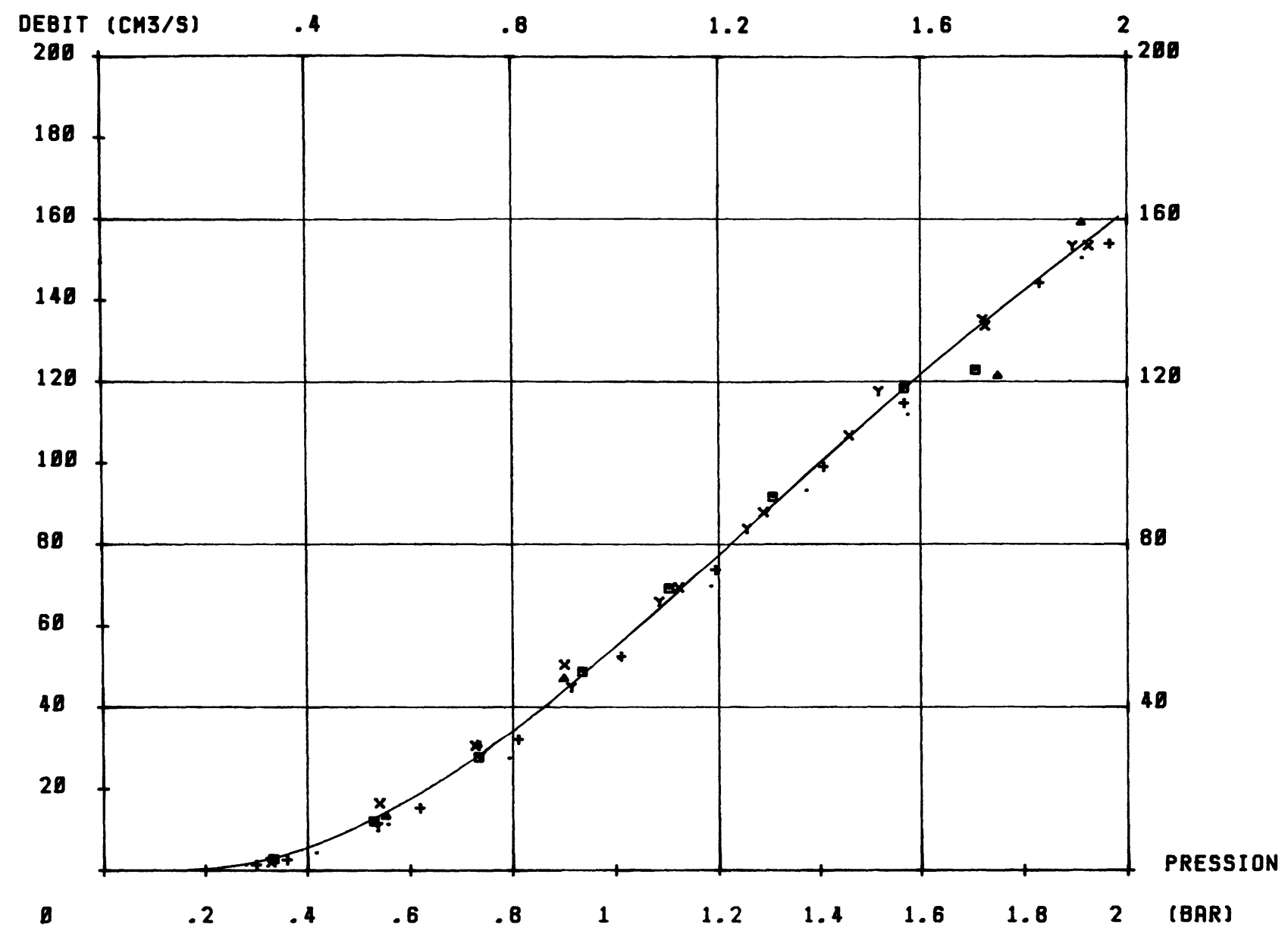

Fig. 6. - Courbe débit/pression.

[Flow/pressure curve.]

\section{Résultats.}

Les tableaux II.1-6 montrent les résultats obtenus.

Nous avons introduit les valeurs moyennes des débits : $\bar{Q}_{\mathrm{v}}$, des levées : $\bar{h}$, déduites des résultats expérimentaux, dans l'expression du nombre de Reynolds $\operatorname{Re}$, de $R_{\mathrm{p}}$, de $X$ et $Y$ (Eq. (16)).

Le calcul des nombres de Reynolds locaux montre que l'écoulement reste toujours laminaire, puisque ceux-ci ne dépassent pas la cinquantaine.

Nous pouvons constater d'autre part, que les nombres adimensionnels $R_{\mathrm{p}}$ et $\boldsymbol{R}_{\mathrm{v}}$ sont proches de l'unité pour les plus hautes pressions que nous ayons eues à utiliser. Nous sommes alors dans la zone prévue par l'analyse phénoménologique où effets visqueux et effets inertiels sont de même importance. Ces deux termes augmentent ensuite lorsque la pression et le débit diminuent. L'influence de la viscosité devient alors prépondérante.

Le calcul de $X$ et $Y$ (Eq. (12)) conduit à la détermination de $\alpha$ et de $\beta$ dont les valeurs sont montrées tableau III. Ces résultats ont été établis pour des clapets amont à quatre orifices, l'épaisseur du clapet aval étant variable.

Les coefficients $\alpha$ et $\beta$ ont été divisés respectivement par $n \ln \left(r_{1} / r_{0}\right)$ et $2 e^{2} n^{2}\left(1 / r_{1}^{2}-1 / r_{0}^{2}\right)$. On peut ainsi les comparer à ceux calculés pour un écoulement se produisant entre deux disques parallèles. On constate que ces coefficients varient avec l'épaisseur des clapets. En effet, ce paramètre intervient dans l'amplitude de déformation de ces derniers.

Les coefficients issus de l'écoulement entre deux disques parallèles sont compris dans la plage de variation donnée plus haut.

Les colonnes 9 et 10, tableaux II.1-6, permettent de comparer l'importance des forces visqueuses et inertielles par application des équations (12) et (13). En colonne 11 est calculée la force de pression statique qui s'exerce au droit de l'orifice.

L'ensemble des essais concernant des clapets aval d'épaisseurs variant de 0,2 à $0,6 \mathrm{~mm}$, soulevés par un nombre de points de poussée variables (Fig. 5) montrent que cette force augmente avec cette épais- 


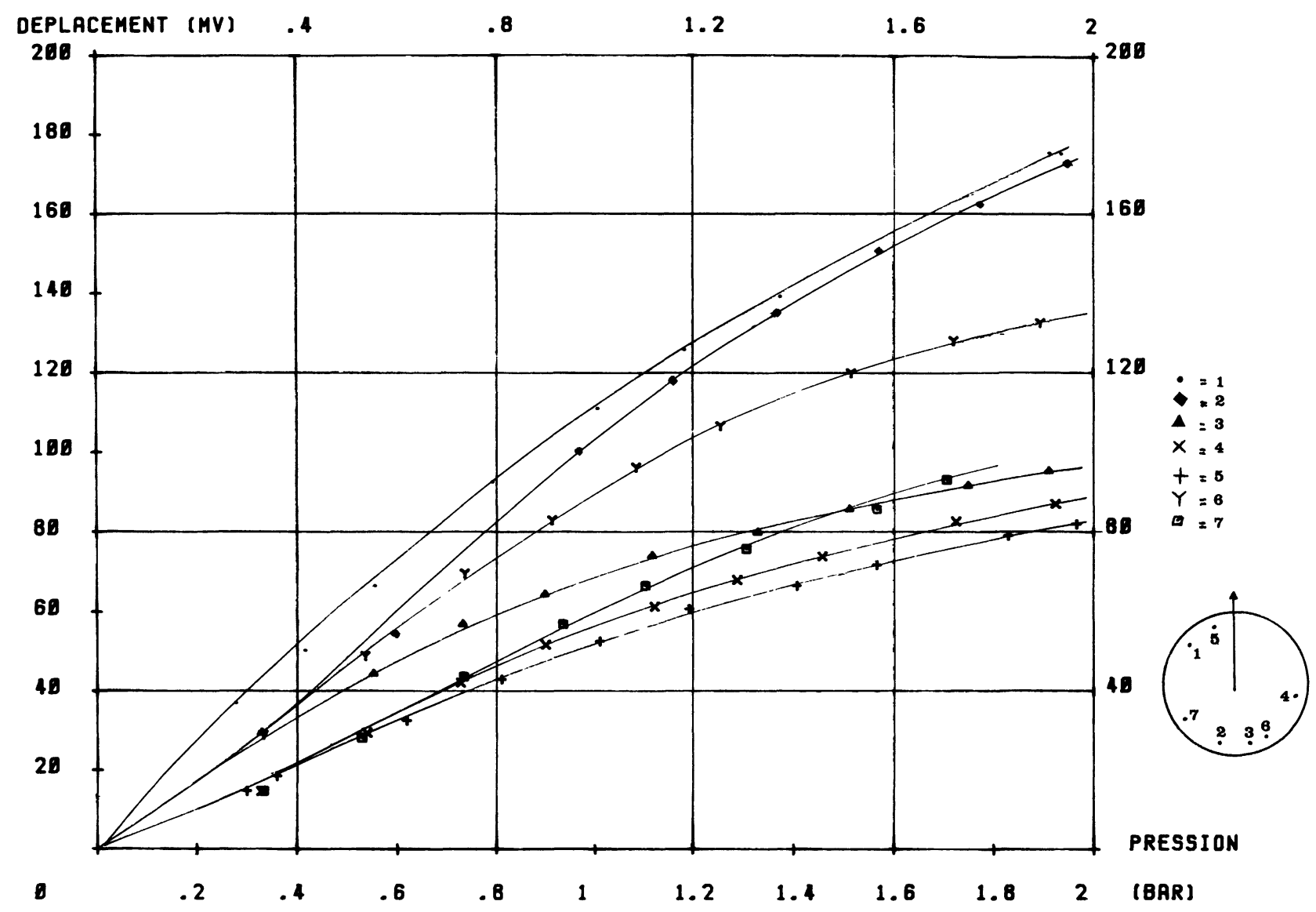

Fig. 7. - Courbe déformation/pression.

[Deformation/pressure curve.]

\section{DIAGRAMME PERIPHERIQUE}

Clapet superieur : épaisseur $0.2-0.6 \mathrm{~mm}$

Clapet inférieur : épaisseur $0.2 \mathrm{~mm}$

4 orifices de poussées

$h e^{3} / r^{4}$

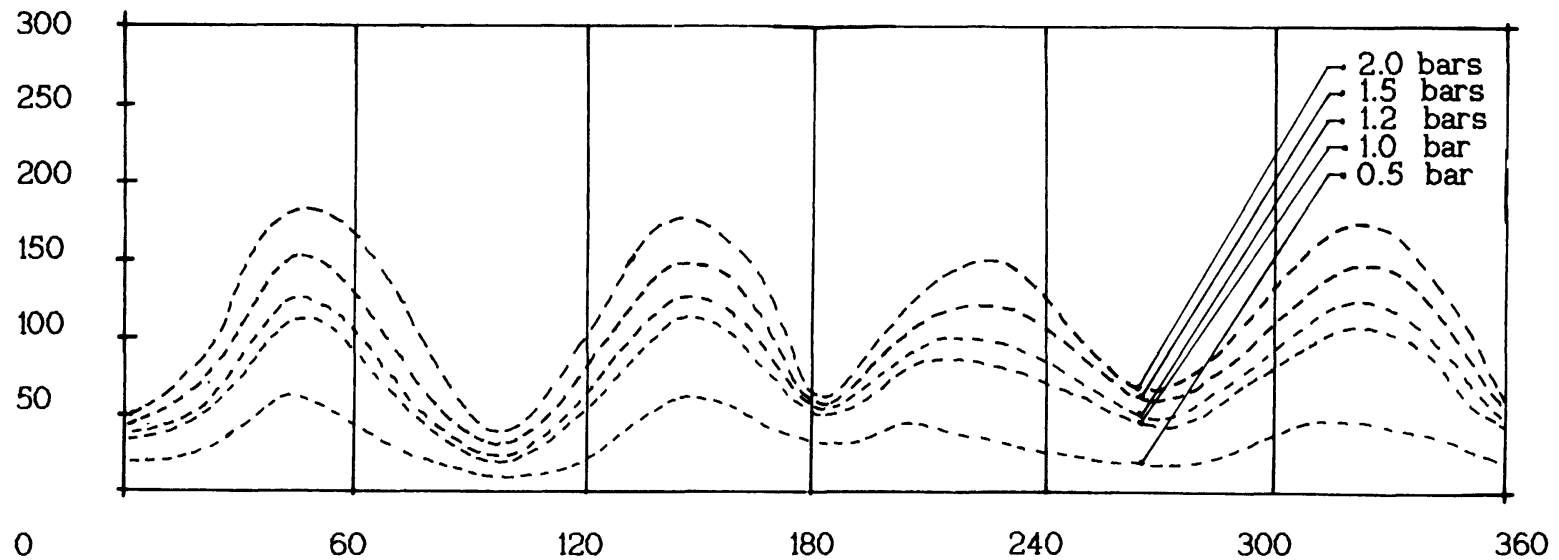

$\Theta$

Fig. 8. - Diagramme périphérique.

[Peripheric diagramme.] 
Tableau II. - Calcul des nombres adimensionnels et des forces à partir des données expérimentales pour différents clapets.

[Dimensionless numbers and forces calculation from experimental datas for different types of valve.]

Tableau II.1.

épaisseur clapet : $0.2 \mathrm{~mm}$

nu $=34$ cts $\quad a=0.153 \quad b=0.0003$

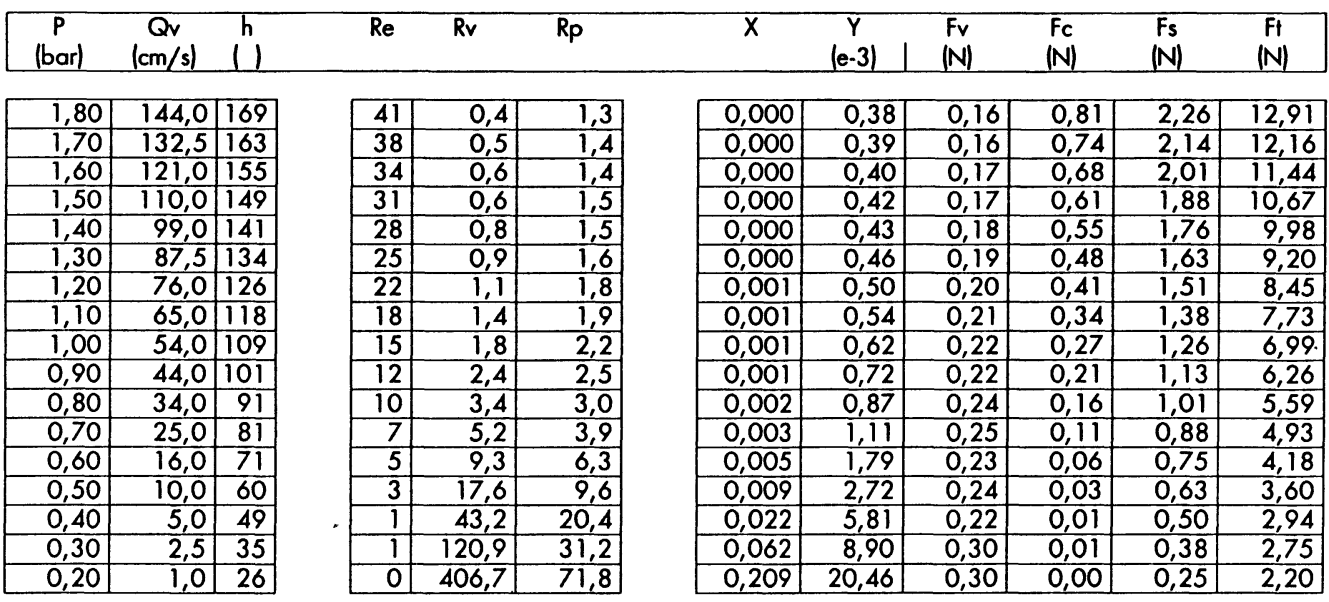

Tableau II.2.

épaisseur clapet : $0.25 \mathrm{~mm}$

nu $=40$ cts $\quad a=0.284 \quad b=0.0014$

\begin{tabular}{|c|c|c|c|c|c|c|c|c|c|c|c|}
\hline $\begin{array}{c}P \\
\text { (bar) }\end{array}$ & $\begin{array}{c}\mathrm{Qv}_{\mathrm{v}} \\
(\mathrm{cm} / \mathrm{s})\end{array}$ & $1^{h}$ & $\overline{\operatorname{Re}}$ & Rv & $R p$ & $x$ & $\begin{array}{c}Y \\
(e-3)\end{array}$ & $\begin{array}{l}\text { Fv } \\
(\mathrm{N})\end{array}$ & $\begin{array}{l}\mathrm{Fc} \\
(\mathrm{N})\end{array}$ & $\begin{array}{c}\text { Fs } \\
(N)\end{array}$ & $\begin{array}{c}F t \\
(N)\end{array}$ \\
\hline 1,80 & 62,0 & 124 & 15 & 1,6 & 3,8 & 0,001 & 1,70 & 0,37 & 0,78 & 2,26 & 13,66 \\
\hline 1,70 & 53,0 & 117 & 13 & 2,0 & 4,4 & 0,002 & 1,96 & 0,38 & 0,64 & 2,14 & 12,62 \\
\hline 1,60 & 45,0 & 110 & 11 & 2,5 & 5,1 & 0,002 & 2,26 & 0,38 & 0,52 & 2,01 & 11,68 \\
\hline 1,50 & 37,5 & 103 & 9 & 3,2 & 6,0 & 0,003 & 2,68 & 0,39 & 0,41 & 1,88 & 10,76 \\
\hline 1,40 & 30,5 & 95 & 7 & 4,3 & 7,2 & 0,003 & 3,21 & 0,40 & 0,32 & 1,76 & 9,95 \\
\hline 1,30 & 23,5 & 88 & 6 & 6,0 & 9,7 & 0,005 & 4,31 & 0,39 & 0,22 & 1,63 & 9,00 \\
\hline 1,20 & 17,5 & 79 & 4 & 9,0 & 13,0 & 0,007 & 5,78 & 0,40 & 0,15 & 1,51 & 8,26 \\
\hline 1,10 & 13,0 & 72 & 3 & 13,3 & 17,9 & 0,011 & 7,98 & 0,40 & 0,10 & 1,38 & 7,52 \\
\hline 1,00 & 9,0 & 64 & 2 & 21,6 & 26,8 & 0,017 & 11,95 & 0,39 & 0,06 & 1,26 & 6,84 \\
\hline 0,90 & 6,0 & 55 & 1 & 37,7 & 40,1 & 0,030 & 17,88 & 0,41 & 0,04 & 1,13 & 6,31 \\
\hline 0,80 & 3,5 & 45 & 1 & 79,0 & 70,2 & 0,063 & 31,26 & 0,44 & 0,02 & 1,01 & 5,85 \\
\hline 0,70 & 2,0 & 36 & 0 & 172,8 & 120,4 & 0,139 & 53,62 & 0,49 & 0,01 & 0,88 & 5,51 \\
\hline 0,60 & 1,0 & 27 & 0 & 460,8 & 232,2 & 0,370 & 103,40 & 0,58 & 0,00 & 0,75 & 5,35 \\
\hline 0,50 & 0,5 & 19 & 0 & 1309,5 & 383,2 & 1,053 & 170,69 & 0,83 & 0,00 & 0,63 & 5,84 \\
\hline
\end{tabular}

Tableau II.3.

épaisseur clapet : $0.3 \mathrm{~mm}$

nu $=39$ cts $\quad a=0.295 \quad b=0.0018$

\begin{tabular}{|c|c|c|c|c|c|c|c|c|c|c|c|}
\hline $\begin{array}{c}P \\
\text { (bar) }\end{array}$ & $\begin{array}{c}Q_{v} \\
(\mathrm{~cm} / \mathrm{s})\end{array}$ & $\|^{h}$ & $\operatorname{Re}$ & Rv & $R p$ & $\bar{x}$ & $\begin{array}{c}Y \\
(e-3)\end{array}$ & $\begin{array}{l}\mathrm{Fv} \\
(\mathrm{N})\end{array}$ & $\begin{array}{l}\mathrm{Fc} \\
(\mathrm{N})\end{array}$ & $\begin{array}{l}\text { Fs } \\
(N)\end{array}$ & $\begin{array}{c}\mathrm{Ft} \\
(\mathrm{N})\end{array}$ \\
\hline 2,20 & 43,0 & 94 & 11 & 3,0 & 5,6 & 0,003 & 3,58 & 0,60 & 0,58 & 2,76 & 15,78 \\
\hline 2,10 & 35,5 & 89 & 9 & 3,8 & 7,0 & 0,004 & 4,49 & 0,58 & 0,44 & 2,64 & 14,65 \\
\hline 2,00 & 35,5 & 85 & 9 & 4,0 & 6,1 & 0,005 & 3,90 & 0,67 & 0,49 & 2,51 & 14,67 \\
\hline 1,90 & 25,5 & 79 & 6 & 6,0 & 9,7 & 0,007 & 6,21 & 0,60 & 0,29 & 2,39 & 13,10 \\
\hline 1,80 & 21,0 & 73 & 5 & 7,9 & 11,5 & 0,009 & 7,40 & 0,62 & 0,23 & 2,26 & 12,46 \\
\hline 1,70 & 17,0 & 69 & 4 & 10,3 & 14,9 & 0,012 & 9,53 & 0,60 & 0,17 & 2,14 & 11,61 \\
\hline 1,60 & 14,0 & 64 & 3 & 13,5 & 17,7 & 0,016 & 11,38 & 0,62 & 0,13 & 2,01 & 11,04 \\
\hline 1,50 & 11,0 & 58 & 3 & 19,0 & 22,1 & 0,022 & 14,20 & 0,65 & 0,10 & 1,88 & 10,54 \\
\hline 1,40 & 8,5 & 53 & 2 & 26,9 & 28,9 & 0,031 & 18,53 & 0,66 & 0,07 & 1,76 & 996 \\
\hline 1,30 & 6,0 & 48 & 1 & 42,1 & 44,2 & 0,049 & 28,32 & 0,63 & 0,04 & 1,63 & 9,21 \\
\hline 1,20 & 4,5 & 43 & 1 & 62,7 & 58,2 & 0,073 & 37,30 & 0,65 & 0,03 & 1,51 & 8,76 \\
\hline 1,10 & 3,0 & 37 & 1 & 109,3 & 88,8 & 0,126 & 56,96 & 0,68 & 0,02 & 1,38 & 8,33 \\
\hline 1,00 & 1,5 & 33 & 0 & 245,0 & 256,9 & 0,284 & 164,77 & 0,48 & 0,01 & 1,26 & 6,97 \\
\hline 0,90 & 1,0 & 28 & 0 & 433,2 & 374,5 & 0,501 & 240,20 & 0,52 & 0,00 & 1,13 & 6,64 \\
\hline 0,80 & 0,5 & 22 & 0 & 1102,7 & 822,0 & 1,276 & 527,25 & 0,54 & 0,00 & 1,01 & 6,19 \\
\hline
\end{tabular}


Tableau II.4.

épaisseur clapel : $0.35 \mathrm{~mm}$

$\mathrm{nu}=38 \mathrm{cts} \quad \mathrm{a}=0.419 \quad \mathrm{~b}=0.0034$

\begin{tabular}{|c|c|c|c|c|c|c|c|c|c|c|c|}
\hline $\begin{array}{c}P \\
\text { (bar) }\end{array}$ & $\begin{array}{c}Q_{v} \\
(\mathrm{~cm} / \mathrm{s} \mid\end{array}$ & il & $\operatorname{Re}$ & Rv & $R p$ & $\bar{X}$ & $\begin{array}{c}Y \\
(e-3)\end{array}$ & $\begin{array}{l}F v \\
(N)\end{array}$ & $\begin{array}{l}F c \\
(N)\end{array}$ & $\begin{array}{l}\text { Fs } \\
\text { (N) }\end{array}$ & $\begin{array}{l}\mathrm{Ft} \\
\text { (N) }\end{array}$ \\
\hline
\end{tabular}

\begin{tabular}{|r|r|r|}
\hline 3,75 & 50,6 & 93 \\
\hline 3,60 & 44,4 & 89 \\
\hline 3,45 & 38,1 & 85 \\
\hline 3,30 & 33,1 & 81 \\
\hline 3,15 & 28,1 & 76 \\
\hline 3,00 & 23,1 & 71 \\
\hline 2,85 & 19,4 & 68 \\
\hline 2,70 & 15,9 & 63 \\
\hline 2,55 & 12,5 & 58 \\
\hline 2,40 & 9,7 & 54 \\
\hline 2,25 & 7,5 & 49 \\
\hline 2,10 & 5,6 & 43 \\
\hline 1,95 & 4,1 & 39 \\
\hline 1,80 & 2,8 & 34 \\
\hline 1,65 & 1,9 & 29 \\
\hline 1,50 & 1,3 & 25 \\
\hline
\end{tabular}

\begin{tabular}{|r|r|r|}
\hline 13 & 2,5 & 6,7 \\
\hline 11 & 3,0 & 7,7 \\
\hline 10 & 3,6 & 9,1 \\
\hline 8 & 4,4 & 10,5 \\
\hline 7 & 5,5 & 12,2 \\
\hline 6 & 7,2 & 15,0 \\
\hline 5 & 9,0 & 18,6 \\
\hline 4 & 11,8 & 22,5 \\
\hline 3 & 16,3 & 29,1 \\
\hline 2 & 22,6 & 39,5 \\
\hline 2 & 32,2 & 51,0 \\
\hline 1 & 49,1 & 65,7 \\
\hline 1 & 73,9 & 93,6 \\
\hline 1 & 124,1 & 140,9 \\
\hline 0 & 214,5 & 204,0 \\
\hline 0 & 363,7 & 294,4 \\
\hline
\end{tabular}

\begin{tabular}{|r|r|r|r|r|r|}
\hline 0,004 & 5,87 & 1,00 & 1,15 & 4,71 & 27,45 \\
\hline 0,005 & 6,70 & 1,00 & 0,96 & 4,52 & 25,97 \\
\hline 0,006 & 7,96 & 0,99 & 0,78 & 4,34 & 24,41 \\
\hline 0,007 & 9,16 & 0,99 & 0,65 & 4,15 & 23,15 \\
\hline 0,009 & 10,68 & 1,02 & 0,53 & 3,96 & 22,04 \\
\hline 0,011 & 13,13 & 1,03 & 0,41 & 3,77 & 20,84 \\
\hline 0,014 & 16,22 & 0,98 & 0,32 & 3,58 & 19,52 \\
\hline 0,019 & 19,64 & 1,01 & 0,25 & 3,39 & 18,62 \\
\hline 0,026 & 25,44 & 1,02 & 0,18 & 3,20 & 17,63 \\
\hline 0,036 & 34,46 & 0,98 & 0,13 & 3,02 & 16,49 \\
\hline 0,051 & 44,50 & 1,02 & 0,09 & 2,83 & 15,74 \\
\hline 0,077 & 57,37 & 1,12 & 0,07 & 2,64 & 15,31 \\
\hline 0,116 & 81,75 & 1,10 & 0,04 & 2,45 & 14,38 \\
\hline 0,196 & 122,98 & 1,14 & 0,03 & 2,26 & 13,70 \\
\hline 0,338 & 178,11 & 1,24 & 0,02 & 2,07 & 13,33 \\
\hline 0,573 & 257,05 & 1,33 & 0,01 & 1,88 & 12,89 \\
\hline
\end{tabular}

Tableau II.5.

épaisseur dapet : $0.4 \mathrm{~mm}$

$\mathrm{nu}=38 \mathrm{cts} \quad \mathrm{o}=0.430$

$b=0.0036$

\begin{tabular}{|c|c|c|c|c|c|c|c|c|c|c|c|}
\hline $\begin{array}{c}P \\
\text { (bar) }\end{array}$ & $\begin{array}{c}Q_{v} \\
(\mathrm{~cm} / \mathrm{s} \mid\end{array}$ & 11 & $\operatorname{Re}$ & $\overline{R v}$ & $R p$ & $\bar{x}$ & $\begin{array}{c}Y \\
(e-3)\end{array}$ & $\begin{array}{l}F_{v} \\
(N)\end{array}$ & $\begin{array}{l}\mathrm{Fc} \\
(\mathrm{N})\end{array}$ & $\begin{array}{l}\text { Fs } \\
\text { (N) }\end{array}$ & $\begin{array}{l}\mathrm{Ft} \\
(\mathrm{N})\end{array}$ \\
\hline 5,40 & 77,0 & 97 & 20 & 1,6 & 4,5 & 0,003 & 5,19 & 1,38 & 1,98 & 6,79 & 40,59 \\
\hline 5,10 & 66,0 & 93 & 17 & 1,9 & 5,4 & 0,004 & 6,13 & 1,34 & 1,58 & 6,41 & 37,34 \\
\hline 4,80 & 56,0 & 87 & 14 & 2,4 & 6,1 & 0,005 & 7,01 & 1,39 & 1,30 & 6,03 & 34,90 \\
\hline 4,50 & 47,0 & 81 & 12 & 3,1 & 7,1 & 0,006 & 8,09 & 1,45 & 1,06 & 5,65 & 32,64 \\
\hline 4,20 & 37.0 & 76 & 9 & 4,2 & 9,4 & 0,009 & 10,72 & 7,38 & 0,74 & $=5,28$ & 29,61 \\
\hline 3,90 & 29,0 & 70 & 7 & 5,8 & 12,1 & 0,012 & 13,75 & 1,38 & 0,54 & 4,90 & 27,30 \\
\hline 3,60 & 21,0 & 64 & 5 & 8,8 & 17,7 & 0,018 & 20,24 & 1,31 & 0,34 & 4,52 & 24,69 \\
\hline 3,30 & 16,0 & 59 & 4 & 12,5 & 23,8 & 0,026 & 27,16 & 1,28 & 0,23 & 4,15 & 22,61 \\
\hline 3,00 & 11,0 & 53 & 3 & 20,3 & 37,0 & 0,042 & 42,15 & 1,21 & 0,14 & 3,77 & 20,46 \\
\hline 2,70 & 8,0 & 47 & 2 & 31,4 & 49,5 & 0,065 & 56,40 & 1,26 & 0,09 & 3,39 & 18,98 \\
\hline 2,40 & 4,0 & 41 & 1 & 72,1 & 133,8 & 0,148 & 152,60 & 0,95 & 0,03 & 3,02 & 15,98 \\
\hline 2,10 & 3,0 & 34 & 1 & 115,9 & 143,2 & 0,238 & 163,24 & 1,25 & 0,02 & 2,64 & 15,65 \\
\hline 1,80 & 2,0 & 28 & 1 & 211,0 & 187,3 & 0,434 & 213,51 & 1,49 & 0,02 & 2,26 & 15,08 \\
\hline 1,50 & 1,0 & 22 & 0 & 537,2 & 385,3 & 1,105 & 439,38 & 1,54 & 0,01 & 1,88 & 13,71 \\
\hline 1,20 & 0,5 & 16 & 0 & 1477,3 & 652,2 & 3,040 & 743,67 & 2,00 & 0,00 & 1,51 & 14,04 \\
\hline
\end{tabular}

Tableau II.6.

épaisseur clapet : $0.6 \mathrm{~mm}$

nu $=43 \mathrm{cls} \quad \mathrm{a}=0.784 \quad b=0.0175$

\begin{tabular}{|c|c|c|c|c|c|c|c|c|c|c|c|}
\hline $\begin{array}{c}P \\
\text { (bar) }\end{array}$ & $\begin{array}{c}\mathrm{Qv}_{\mathrm{v}} \\
(\mathrm{cm} / \mathrm{s})\end{array}$ & 11 & $\operatorname{Re}$ & $R v$ & $R p$ & $\bar{x}$ & $\begin{array}{c}Y \\
(e-3) \\
\end{array}$ & $\begin{array}{l}\mathrm{Fv} \\
(\mathrm{N})\end{array}$ & $\begin{array}{l}\mathrm{Fc} \\
(\mathrm{N})\end{array}$ & $\begin{array}{l}\mathrm{Fs} \\
(\mathrm{N})\end{array}$ & $\begin{array}{l}\mathrm{Ft} \\
(\mathrm{N})\end{array}$ \\
\hline 12,50 & 55,0 & 79 & 12 & 3,1 & 13,7 & 0,014 & 35,12 & 3,77 & 3,29 & 15,71 & 91,06 \\
\hline 12,00 & 47,0 & 75 & 11 & 3,8 & 16,2 & 0,018 & 41,61 & 3,76 & 2,67 & 15,08 & 86,03 \\
\hline 11,50 & 39,5 & 70 & 9 & 4,8 & 19,2 & 0,022 & 49,18 & 3,89 & 2,16 & 14,45 & 82,01 \\
\hline 11,00 & 33,0 & 67 & 7 & 6,0 & 24,1 & 0,028 & 61,74 & 3,71 & 1,65 & 13,82 & 76,70 \\
\hline 10,50 & 27,0 & 64 & 6 & 7,7 & 31,3 & 0,036 & 80,33 & 3,48 & 1,21 & 13,19 & 71,52 \\
\hline 10,00 & 22,0 & 59 & 5 & 10,3 & 38,2 & 0,048 & 97,94 & 3,62 & 0,94 & 12,57 & 68,51 \\
\hline 9,50 & 18,0 & 55 & 4 & 13,5 & 47,1 & 0,063 & 120,78 & 3,65 & 0,73 & 11,94 & 65,27 \\
\hline 9,00 & 15,0 & 51 & 3 & 17,5 & 55,2 & 0,081 & 141,67 & 3,82 & 0,59 & 11,31 & 62,86 \\
\hline 8,50 & 11,5 & 47 & 3 & 24,7 & 75,2 & 0,115 & 193,33 & 3,74 & 0,41 & 10,68 & 59,31 \\
\hline 8,00 & 9,0 & 43 & 2 & 34,6 & 96,9 & 0,160 & 248,67 & 3,82 & 0,30 & 10,05 & 56,69 \\
\hline 7,50 & 7,0 & 39 & 2 & 49,0 & 123,6 & 0,227 & 317,01 & 3,98 & 0,22 & 9,42 & 54,51 \\
\hline 7,00 & 5,5 & 34 & 1 & 71,5 & 142,0 & 0,331 & 364,26 & 4,73 & 0,18 & 8,80 & 54,80 \\
\hline 6,50 & 4,0 & 29 & 1 & 115,3 & 181,3 & 0,534 & 465,23 & 5,54 & 0,13 & 8,17 & 55,34 \\
\hline 6,00 & 3,0 & 25 & 1 & 178,3 & 221,2 & 0,826 & 567,38 & 6,48 & 0,10 & 7,54 & 56,49 \\
\hline 5,50 & 2,5 & 21 & $i$ & 254,7 & 206,0 & 1,179 & 528,45 & 9,12 & 0,10 & 6,91 & 64,50 \\
\hline 5,00 & 2,0 & 17 & 0 & 393,3 & 191,7 & 1,821 & 491,91 & 13,75 & 0,09 & 6,28 & 80,50 \\
\hline 4,50 & 1,5 & 15 & 0 & 594,4 & 238,8 & 2,752 & 612,77 & 15,01 & 0,07 & 5,65 & 82,93 \\
\hline 4,00 & 1,0 & 13 & 0 & 1028,8 & 358,8 & 4,763 & 920,51 & 15,37 & 0,04 & 5,03 & 81,75 \\
\hline
\end{tabular}


Tableau III. - Coefficients expérimentaux de la régression linéaire pour différents clapets. [Experimental coefficients of the linear regression for different types of valve.]

\begin{tabular}{|c|llllll|}
\hline Epaisseurs clapets & $0,20 \mathrm{~mm}$ & $0,25 \mathrm{~mm}$ & $0,30 \mathrm{~mm}$ & $0,35 \mathrm{~mm}$ & $0,40 \mathrm{~mm}$ & $0,60 \mathrm{~mm}$ \\
\hline$\alpha / n \ln \left(r_{1} / r_{0}\right)$ & 0,10 & 0,17 & 0,18 & 0,26 & 0,26 & 0,47 \\
$\beta / 2 e^{2} n^{2}\left(r_{1}^{-2}-r_{0}^{-2}\right)$ & 0,0018 & 0,0050 & 0,0045 & 0,0062 & 0,0051 & 0,0109 \\
$r^{2}$ & 0,999 & 0,993 & 0,995 & 0,996 & 0,992 & 0,992 \\
\hline
\end{tabular}

seur et avec ce nombre de points. Elle peut atteindre jusqu'à $90 \mathrm{~N}$ dans le cas où le clapet a une épaisseur de $0,6 \mathrm{~mm}$.

\section{Conclusion.}

Le calcul des nombres de Reynolds, toujours inférieurs à 50 en cet endroit, montre que l'écoulement reste laminaire dans l'entrefer. On peut, d'autre part, constater une bonne corrélation entre ce qu'avaient prévu les calculs et ce que donnent les essais.
De ces derniers, nous déduisons, en comparant les termes visqueux et inertiels que l'écoulement se produit principalement en régime visqueux $\left(R_{\mathrm{v}}>1\right.$ et $R_{\mathrm{v}} \sim R_{\mathrm{p}}$ ), même si la zone de transition est quelquefois atteinte. Cependant, dans le cas d'un amortisseur en fonctionnement réel, les vitesses de tige et donc les vitesses du fluide sont plus élevées. Il est donc probable que le régime inertiel est atteint. En conséquence, les pertes de charge devraient être fonction de la température en basse vitesse de tige lorsque le régime est visqueux. Inversement, elles en seront indépendantes pour de grandes vitesses de tige.

\section{Bibliographie}

[1] AVNER D., L'amortisseur et son influence sur la suspension automobile (SIA 5/73) pp. 367-374.

[2] BERTHOz A., Effets biomécaniques et physiologiques des vibrations sur l'homme (SIA 6/67) pp. 323336.

[3] Lavauzelle Ch., Traité de technique automobile, fascicule 12 (1953) pp. 53-72.

[4] Allinguant, Principe de fonctionnement de l'amortisseur, Rapport interne 5/85.

[5] Kubilay E., Pertes d'énergie et levées du clapet au niveau du piston d'un amortisseur hydraulique, Rapport de travail (1983) LMEF, pp. 1-19.

[6] Sekiguchi H., Asami T., Fundamental investigation of an oil damper, First report. Case of its analysis as steady flow, JSME (1982) Vol. 25, pp. 1135-1142.

[7] ERbER, Manomètres et Capteurs (Masson) 1982, pp. 31-63.
[8] Chen Che Pen, Contribution à l'étude de l'écoulement radial d'un fluide visqueux. J. Méc. 5 (1966) 245-259.

[9] Comolet R., Ecoulement d'un fluide entre deux plans parallèles. Contribution à l'étude des butées d'air. Publ. sci. tech. Minist. Air 334 (1957) 23-50.

[10] Moller P. S., Radial flow without swirl between parallel discs. The Aeronautical quaterly (1963) pp. 163-185.

[11] Peube J. L., Pecheux J., Ecoulements d'un fluide visqueux incompressible entre deux plans parallèles fixes, J. Méc. 16 (1977) 170-189.

[12] GinN M., Observations on the radial flow of water between fixed parallel plates, App. Sci. Res. Sect. A 5, pp. 255-264. 